\title{
Multi-Channel Two-way Time of Flight Sensor Network Ranging
}

\author{
Paolo Pettinato, Niklas Wirström, Joakim Eriksson, Thiemo Voigt \\ \{paolope, niwi, joakime, thiemo\}@sics.se \\ Swedish Institute of Computer Science
}

\begin{abstract}
Two-way time of flight (ToF) ranging is one of the most interesting approaches for localization in wireless sensor networking since previous ToF ranging approaches using commercial off-the-shelf (COTS) devices have achieved good accuracy. The COTS-based approaches were, however, evaluated only in line-of-sight conditions. In this paper, we extend ToF ranging using multiple IEEE 802.15.4 channels. Our results demonstrate that with multiple channels we can achieve good accuracy even in non line-of-sight conditions. Furthermore, our measurements suggest that the variance between different channels serves as a good estimate of the accuracy of the measurements, which can be valuable information for applications that require localization information.
\end{abstract}

\section{Introduction}

Localization of sensor nodes is an important problem, because sensor data is in many cases useless without the knowledge about the location from where it was obtained. Therefore a lot of effort has been devoted to solve the localization problem $[6,12,13,17]$. There is, however, still no generic solution, i.e., a solution that is also accurate when nodes are not in line-of-sight (LoS), for commercial off-the-shelf (COTS) devices. The localization problem can be divided into two sub-problems: collection of localization measurements, and computation of the actual positions based on the collected measurements. In this paper we focus on the former.

Our key idea to improve the accuracy of ToF-based ranging when nodes are not in LoS is to use multiple channels for estimating the time of flight. We present experiments that show that picking one channel only, can lead to very inaccurate results. For larger distances of about 50 meters the difference between estimations with the best and the worst channel can differ by up to 20 meters. Our main contribution is that we demonstrate that using multiple channels enables us to achieve good ranging accuracy also when nodes are not placed in LoS of each other. Under non-LoS conditions, the multi-path effect can be substantial which can lead to longer ToF measurements than required for the actual distance between sender and receiver. Our estimation method that we call the $k$-sigma method compensates for this effect by using the variance between measurements from different channels, i.e., the inter-channel variance. 
In this paper, we extend the two-way time-of-flight (ToF) approach by Mazomenos et al. for relative ranging with COTS devices [14]. Mazomenos et al. have achieved very accurate localization estimations for nodes placed in LoS but have not tackled the problem when nodes are not in LoS. One additional contribution of our paper is that we confirm their LoS results using a hardware platform with the commonly used $\mathrm{CC} 2420$ radio while they used a TI EZ430-RF2500 development platform. Since the CC2420 radio is IEEE 802.15.4 compliant we can make use of hardware acknowledgements which reduces the timing uncertainties when the receiver's software processes the received packet and acknowledges the ranging packet. In the general case, when we have a scenario with both LoS and non LoS, our solution outperforms the one proposed by Mazomenos et al.[14], achieving a 30\% smaller overall root mean square (RMS) estimation error. We also present results that suggest that the inter-channel variance can serve as an estimate for the accuracy of the distance estimation. Such information is useful input for algorithms that compute positions based on distance estimations.

Our results also highlight the advantages of using a stable clock source. Towards this end, we use the radio's clock instead of that of the CPU. We show that this way, variations in temperature affect our measurements to a small extent only. Furthermore, the variance in clock speed between devices is lower which avoids the need for pairwise calibration.

The paper proceeds as follows. In the next section we present two-way time of flight ranging. In Section 3 we present the design and implementation of our system. The following section describes our algorithms for estimating the distance based on ranging measurements of multiple channels. Section 5 presents the experimental results that support our claims. Before concluding we discuss related work in Section 6.

\section{Two-way Time of Flight Ranging}

The basic idea of the ToF ranging is to estimate the distance between two nodes based on the measurements of the propagation time that is linearly correlated to the physical distance between the nodes when nodes are in LoS. Two-way ToF ranging, as opposed to one-way, does not require tight time synchronization between sender and receiver, which is a difficult problem in WSNs [4].

We can compute the distance according to Equation 1 where $c$ is the speed of light, $\hat{t}_{R T T}$ is an average of multiple round-trip-time measurements, and $t_{\text {off }}$ is an offset time accounting for all processing delays in the system. This includes the time for the sender to transmit the packet, the time the receiver needs to process it, and send the acknowledgment.

$$
d=\frac{c}{2}\left(\hat{t}_{R T T}-t_{\text {off }}\right)
$$

From Equation 1 we can derive the following two objectives for designing a two-way ToF ranging system: Accurate measurements of the time of flight 
between pairs of nodes, and that the delay $t_{\text {off }}$ should be as close to a constant as possible. Towards this end, we need a stable clock source with high resolution and precision, efficient time stamping of the ranging packets and predictable execution times.

A single measurement is not sufficient: in fact, the resolution of a clock with frequency $f_{\text {timer }} \mathrm{Hz}$ allows for a spatial precision equal to $\Delta_{d}=\frac{c}{2 \cdot f_{\text {timer }}}$. For a $8 \mathrm{MHz}$ clock, the resulting spatial resolution is $18.737 \mathrm{~m}$. To achieve a better resolution, it is possible to perform a series of measurements and compute the average, a technique also used by Mazomenos et al. [14]. This way, sub-clock precision can be achieved.

\section{System Design and Implementation}

In this section we describe the design and implementation of our ranging system. We use an off-the-shelf sensor node called Z1 from Zolertia [23], a variant of the Tmote Sky. As the Tmote Sky, the Z1 node features an MSP430 processor and an IEEE 802.15.4-compliant CC2420 radio.

\subsection{The Hardware Platform}

The clock source of the timer used to measure the RTT plays a special role. The internal oscillator of the MSP430 processor is a digitally controlled oscillator (DCO) and exhibits a high variance and instability. It is possible to setup the DCO with precalibrated settings at defined nominal frequencies [8]. Since the DCO's frequency depends on temperature and supply voltage, it must, however, be periodically recalibrated against a stable clock source. On our Z1 nodes, we use the $8 \mathrm{MHz}$ internal clock of the radio as a timer source to avoid the need for compensating for clock skew.

The radio clock's signal is generated with a crystal oscillator with a minimum accuracy is $40 \mathrm{ppm}$ [7]. This corresponds to an uncertainty of $\pm 0.32 \mathrm{kHz}$ on the radio clock frequency. This should be compared to that of the MSP430's internal DCO, which is $400 \mathrm{kHz}$ according to the datasheet, or $6.5 \mathrm{kHz}$ according to measurements performed by Mazamenos et al.

Our approach uses a special debug mode of the radio which outputs the radio clock on the Clear Channel Assessment (CCA) pin, and requires the nodes to be slightly modified to route this signal to the timer source input, as shown in Figure 1. This modification can also be applied to a Tmote Sky. This way, we exploit the most stable clock source available on the sensor board. Furthermore, the signals from the radio chip are synchronous with this clock. These signals include the Start of Frame Delimiter (SFD) which enables us to use the radio clock as a timer source to measure time intervals between the SFDs of the sender's ranging packet and the receiver's acknowledgment. When the radio is not used for ranging purposes, the system restores the CCA pin to its normal functionality. 


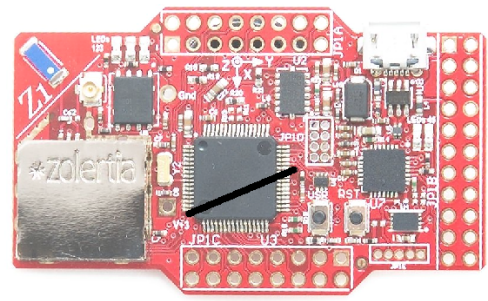

Fig. 1. Hardware modification on the Zolertia Z1. In order to route the clock radio to the MCU timer unit, we use a cable to connect the marked vias.

In order to reduce the uncertainty in software processing on the receiver side, we exploit that the CC2420 radio chip has an automatic acknowledgment feature for packets correctly framed according to the standard. In contrast to Mazomenos et al. who implement the acknowledgement functionality in software [14], we use this hardware acknowledgement mechanism to reduce variability of the delay $t_{o f f}$ in Equation 1.

\subsection{RTT Measurement}

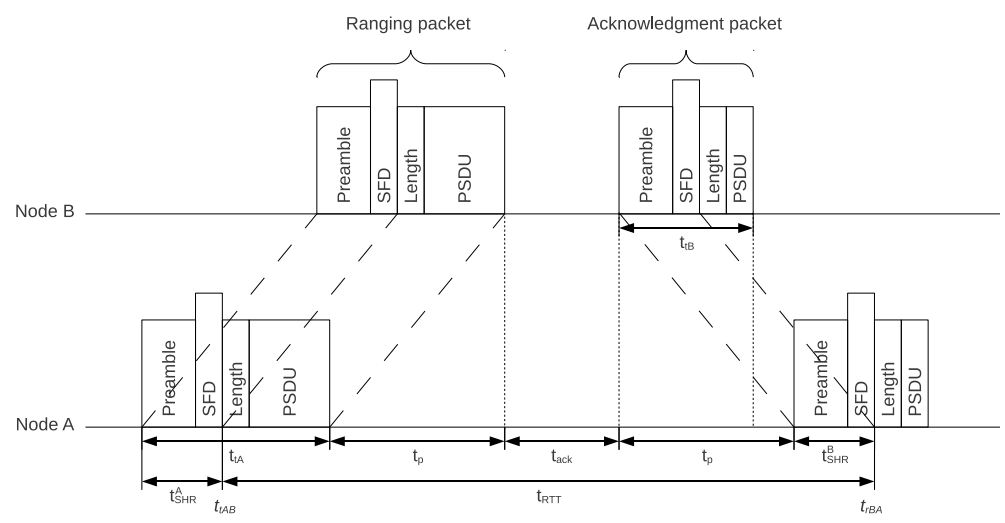

Fig. 2. RTT measurement transaction time-line.

Figure 2 shows the time-line of a single two-way ToF transaction. The detailed process is as follows:

1. Node $A$ prepares a ranging packet with the appropriate "acknowledgment required" attribute set.

2. Node $A$ 's radio sends the ranging packet. After the SFD field is transmitted, the packet is timestamped. The timestamp is stored in $t_{t A B}$. 
3. Node $B$ receives the packet. After $t_{a c k}$, it sends an acknowledgment packet back to node $A$.

4. Node $A$ receives the acknowledgment. As soon as the SFD is decoded, the packet is timestamped and the reception timestamp is stored in $t_{r B A}$.

5. Node $A$ computes the round-trip-time, given by $t_{R T T}=t_{r B A}-t_{t A B}$, and stores it in a buffer.

Figure 2 also shows that:

$$
t_{R T T}=2 t_{p}+t_{t A}+t_{a c k}+t_{S H R}^{B}-t_{S H R}^{A} .
$$

From Equation 1 and Equation 2, we can derive that $t_{\text {off }}$ is composed as follows:

$$
t_{o f f}=t_{t A}+t_{a c k}+t_{S H R}^{B}-t_{S H R}^{A} .
$$

Since we use automatic acknowledgements and an IEEE 802.15.4 radio with a given bandwidth of $250 \mathrm{kbit} / \mathrm{s}$, all constants of Equation 3 are independent of the MCU's DCO, and depend only on the internal crystal oscillator of the radio chip.

The time measurement for the round-trip-time $t_{R T T}$, is obtained from the number of clock cycles $n_{\text {cycles }}$ counted by the timer between the two SFDs.

$$
t_{R T T}=\frac{n_{\text {cycles }}}{f_{\text {timer }}} .
$$

Here, $f_{\text {timer }}$ is the frequency of the radio's internal crystal oscillator. In our case $f_{\text {timer }}=8 \mathrm{MHz}$. As outlined in the previous section we compute $n_{\text {cycles }}$ as the average of several round-trip-time measurements.

\section{Distance Estimation}

In this section we describe our $k$-sigma distance estimation method. It uses interchannel variability of ToF measurements to achieve accurate distance estimation also when nodes are not in LoS. The method uses two parameters that must be calibrated before deployment. We use a calibration method that takes real-world measurements as input to estimate the parameters to be used in subsequent deployments. Note that we do not need to calibrate for each different node or for different pairs of nodes because the deviations in clock speed between different sensor nodes are small, which we confirm in our evaluation in Section 5.

From Equation 1 and Equation 4, we derive that the distance $d$ can be expressed as in Equation 5.

$$
d=\frac{c}{2}\left(\frac{n_{\text {cycles }}}{f_{\text {timer }}}-t_{\text {off }}\right) .
$$

Mazomenos et al. use the average of many short distance measurements to derive the delay $t_{\text {off }}$. We take a different approach, and assume that the offset 
$t_{\text {off }}$ is the sum of a constant processing delay $t_{\text {proc }}$ and a variable delay $t_{m u l t i}$ that is caused by multi-path propagation. See Equation 6 .

$$
t_{\text {off }}=t_{\text {proc }}+t_{\text {multi }} \text {. }
$$

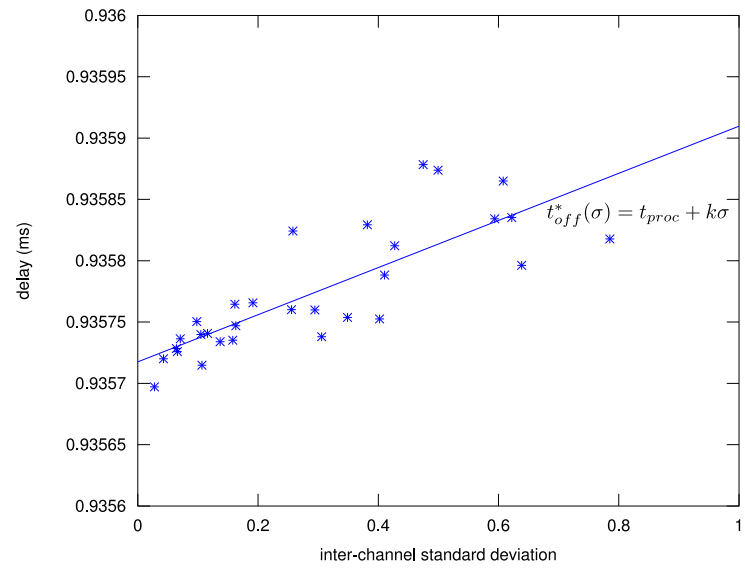

Fig. 3. The relation between the delay $t_{o f f}^{i}$, and the inter-channel standard deviations $\sigma_{i}$ for each measurement $i$ in our data sets. We use this relation to estimate the total delay $t_{o f f}^{*}$ as the sum of the constant delay $t_{\text {proc }}$ caused by processing times, and the variable delay $t_{m u l t i}=k \sigma$ caused by multi-path effects. We use linear regression to estimate the constants $t_{\text {proc }}$ and $k$.

We compute the empirical value of $t_{\text {off }}$ for each measurement $i$ in the data from the experiments presented in Section 5 , as $t_{\text {off }}^{i}=\frac{n_{\text {cycles }}}{f_{\text {timer }}}-2 \frac{d_{i}}{c}$, where $d_{i}$ is the true distance for that measurement, and $n_{\text {cycles }}$ is the average of the measurements from all channels. We also compute the inter-channel standard deviation $\sigma_{i}$ for each measurement. Figure 3 shows the relation between $\sigma_{i}$ and $t_{o f f}^{i}$ for the data. The figure supports the assumption that the offset $t_{o f f}$ can be modeled as the sum of a constant, and a variable delay. It also suggests that we can approximate $t_{\text {off }}$ by a linear function in $\sigma$ :

$$
t_{\text {off }}^{*}(\sigma)=t_{\text {proc }}+t_{\text {multi }}=t_{\text {proc }}+k \sigma .
$$

We use linear regression to estimate the constants $k$ and $t_{\text {proc }}$. These two parameters are the output of our calibration method. The input data is composed of the multi channel ToF measurements together with their corresponding true distances.

The k-sigma method can be optimized for a specific known environment, or for a generic unknown environment. For example, if the deployment target environment is known, and homogeneous in the sense that all nodes have either $\mathrm{LoS}$ or not, we can optimize $k$-sigma for this by using calibration data from a 
similar environment. On the other hand, if the environment is not known, or is mixed such that some nodes have LoS and others do not, it is important that the calibration data come from both LoS and non-LoS situations. This is exemplified in Sections 5.2 through 5.4 .

When nodes are deployed, Equation 8 is used to compute the distance estimation $d^{*}$.

$$
d^{*}=\frac{c}{2}\left(\frac{n_{\text {cycles }}}{f_{\text {timer }}}-t_{\text {proc }}-k \sigma\right)
$$

In Equation 8, we obtain $n_{\text {cycles }}$ and $\sigma$ directly from the measurements. $t_{\text {proc }}$ and $k$ are the values from the calibration phase discussed above.

\section{Experimental Evaluation}

In this section we present experimental results that support the main claims of our paper. First, we evaluate the impact of our choice to use an external, crystal-controlled clock source, rather than the internal clock provided by the DCO. Second, by comparing our results to those of Mazomenos et al., we show that our method provides as accurate results as state-of-the art methods for ToF ranging when nodes are in LoS. Third, we demonstrate the benefit of using multiple channels as opposed to using a single channel, by showing that different channels can give rise to different ToF measurements. Fourth, we present results that show that our method is able to provide accurate estimations even when nodes are not in LoS. Finally, we present results that suggest that we can use the inter-channel standard deviation of the ToF to estimate the accuracy of the distance estimations.

We use data collected from four different scenarios: (1) Outdoors in a field where nodes are positioned in LoS. (2) Outdoors, but with one node on each side of a corner of a brick building in non-LoS. (3) Indoors, in an office corridor with LoS. (4) Indoors, non-LoS around corners, and through walls. We refer to these sets as los-out, nlos-out, los-in, and nlos-in, respectively. For each experiment we perform about 1000 measurements from each of the 16 different 802.15.4 radio channels.

Leave-one-out cross-validation is a well known validation technique often used in the field of Machine Learning [19]. Using this technique, if the data contains $n$ observations, then $n-1$ observations are used to estimate the constants $k$ and $t_{\text {proc }}$ (see Section 4), and the single remaining observation is used to evaluate the accuracy of the estimation. This is then repeated until each observation has been used for evaluation exactly once. All results regarding our estimation method are obtained by using this technique if not mentioned otherwise.

We compare the k-sigma method to that presented by Mazomenos et al., by first estimating $t_{\text {off }}$ using a short-distance calibration measurement. Then we use this value to compute the distance estimations according to Equation 1. In their paper, they also present a filtering method. Their method, however, is not appropriate for our data because our measurements have a much smaller variance. Applying their filter to our measurements results in lower accuracy. 


\subsection{Clock Source Stability}

In the experiments in this section, we analyze the impact of using a stable clock source. As an example of a stable clock source, we use the CC2420's clock which has a signal generated by a $8 \mathrm{MHz}$ crystal-controlled oscillator. We provide experimental results to compare this clock with the digitally-controlled oscillator (DCO) provided by the MCU. The results show that the stable clock provided by the CC2420 radio is a better choice than the DCO, because it enables us to calibrate only once to estimate $t_{\text {proc }}$ and $k$ as discussed in Section 4 , as opposed to calibrating for each pair of nodes. It is also insensitive to temperature changes, and avoids therefore the need for recalibration which would be necessary with the DCO.

RTT distribution. In this experiment we perform several series of RTT measurements, using several pairs of nodes. Nodes are placed at a distance of $15 \mathrm{~cm}$ from one another. We switch the timer clock source between the radio clock and the DCO clock every 512 measurements until a total of 4096 measurements are performed for each source.

Figure 4 shows the results from three different pairs. The DCO measurements have a difference of up to almost 30 clock cycles which would lead to very different estimated distances: according to Equation 5 to more than 500 meters. The corresponding difference for the more stable clock source is 0.02 clock cycles, or $0.40 \mathrm{~m}$, which is a great improvement over the DCO approach. With DCO measurements, it would be necessary to calibrate each node pair in the system to account for clock differences across different nodes. With our approach, this is not necessary.

Moreover, the distributions of the RTTs acquired with the DCO clock are much wider than the ones acquired with the crystal sourced clock. The standard deviation of the former is at least twice the latter. This is also evident in Figure 4 where DCO measurements span across at least 6 different RTT values, while the radio clock measurements span less than the half. This, however, does not necessarily imply that we can obtain more accurate distance estimations with the crystal sourced clock, because one can compensate a low clock stability by increasing the number of RTT measurements.

Temperature drift. In this experiment, we analyze the behavior of the DCO and the CC2420's crystal-controlled oscillator at different temperatures. We place a pair of nodes $15 \mathrm{~cm}$ apart. The nodes sample the temperature using the MCU's internal temperature sensor every 512 RTT measurements. When sampling, we also switch the timer clock source between the radio clock and the DCO clock. We first heat the measuring node and then let it cool down to room temperature. Figure 5 shows the RTTs measured with the two clock sources, in relation to the node temperature. We can see that the effect of the temperature on the RTTs measurements depends on the clock source used. Heating the node affects the DCO measurements heavily, while it has a limited impact on the 

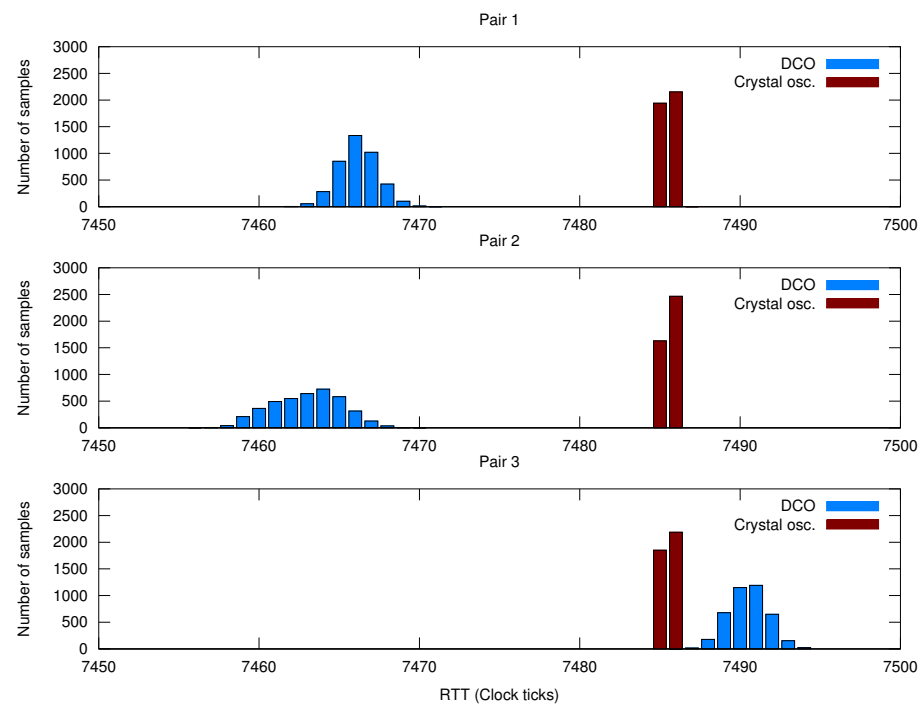

Fig. 4. RTT measurements distributions with DCO and crystal clock source for three pairs of nodes. The low standard deviation of the crystal source avoids the need for pairwise calibration of nodes.

measurements with the stable clock. The difference between the DCO measurements at $30^{\circ} \mathrm{C}$ and $60{ }^{\circ} \mathrm{C}$ is almost 30 clock cycles while the difference across the stable clock measurements is below 0.1 clock cycles. With DCO measurements, we would need to recalibrate the estimation algorithm to take into account the varying temperature. On the other hand, the effects of the temperature on the stable clock are negligible.

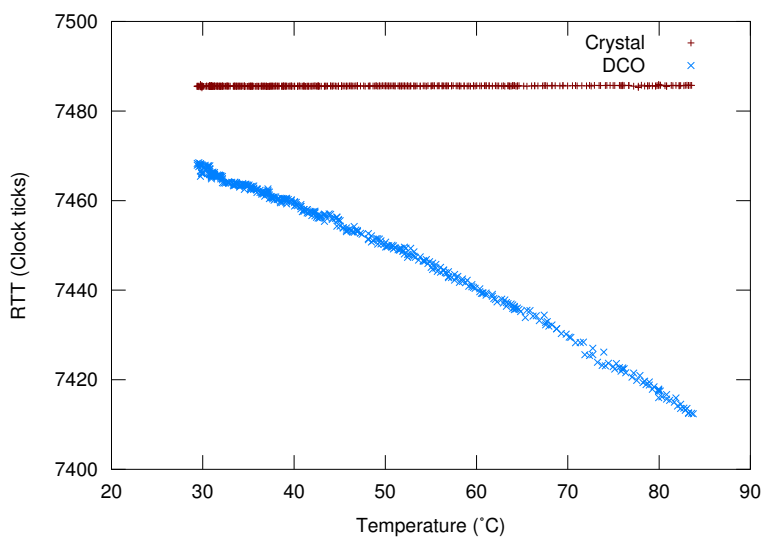

Fig. 5. The impact of the temperature is high on the DCO but low on the crystal oscillator. 


\subsection{A Line-of-Sight Scenario}

We compare the results presented by Mazomenos et al. [14] to those of the $k$ sigma method for LoS measurements. In this experiment we use only the los-out data set as input to the $k$-sigma method for estimating the parameters $t_{\text {proc }}$ and $k$, and hence, in this experiment the calibration environment is similar to the target environment. Note that in this LoS scenario, we do not expect more accurate results with our system than the results they report. First, using the radio's clock improves simplicity and flexibility but not accuracy. Second, our $k$ sigma method targets generic environments rather than scenarios where all nodes are in LoS. The purpose of the experiment is to show that the performance of our method, if optimized for a LoS scenario, is comparable to that of the method proposed by Mazomenos et al.

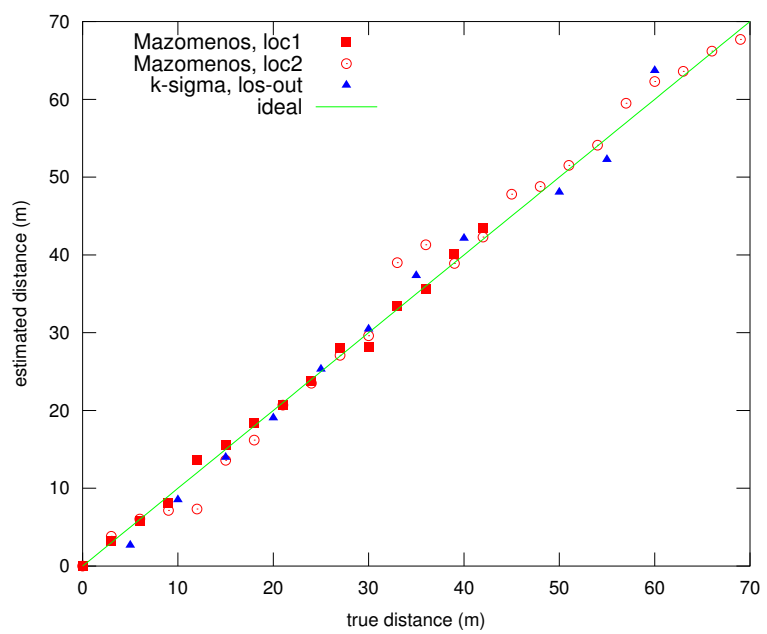

Fig. 6. A comparison of the results presented by Mazomenos et al. and the $k$ sigma method. Here, only the los-out data set is used to estimate the constants $t_{\text {proc }}$ and $k$ for our method. loc1 and loc2 refer to the experiments at Location 1, and Location 2 in their paper [14].

Figure 6 shows the comparison. The values labeled loc1 and loc2 correspond to the outdoor experiments at Location 1 and Location 2 in the paper by Mazomenos et al. At these locations, they obtain RMS errors of 0.75 meter, and 2.23 meter, respectively. We obtain an RMS error of 2.02 meter, which is similar to their results. It should be noted that we are not able to obtain as good results with their method on our data sets, as can be seen in Section 5.4.

\subsection{One Channel is not Enough}

Different channels are subject to different effects caused by multi-path propagation. The variance between measurements from different channels can therefore 
be large. Figure 7 shows an example of this: Circles indicate the estimated distance when we use measurements from single channels for the los-out data set. For some measurements, there is a big difference between the best and the worst single-channel estimation. This is true in particular for longer distances. For example, when the true distance is 50 meters, the estimations differ by more than 20 meters between the worst and the best channel. This indicates that it is not enough to use a single channel, if we want to ensure relatively low estimation errors. One way to improve estimations is by using the smallest value of the different channels. This is indicated by the line that goes through the lower circles in the graph. The k-sigma method, however, produces even better results, as shown in Figure 6, by using the inter-channel standard deviation to estimate the measurement error.

While more measurements increase the accuracy of the estimation, our results show that our improvements stem from the fact that we use multiple channels. We have experimentally verified that using more measurements would not lead to such improvements.

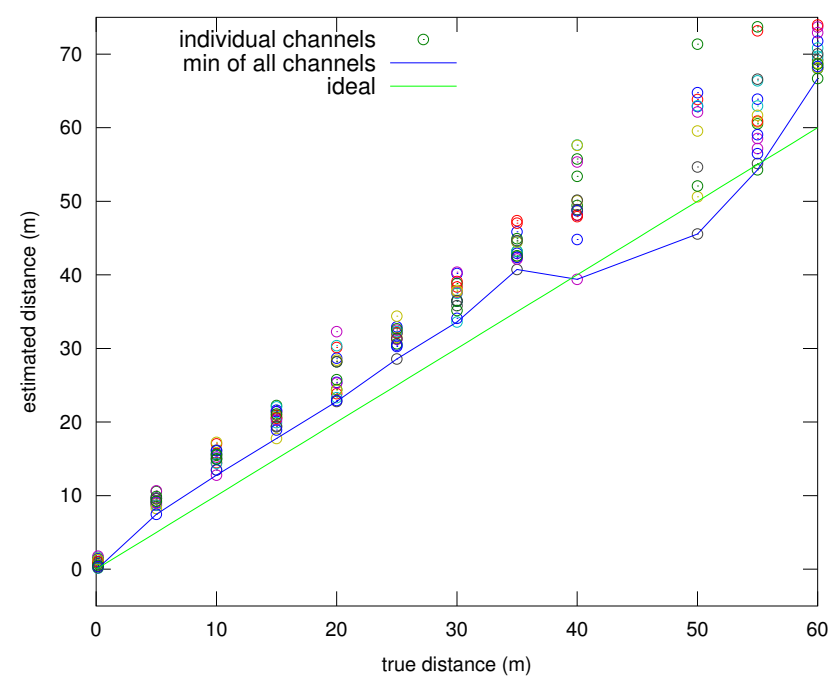

Fig. 7. One channel is not enough. Circles indicate estimated distance for an individual channel. Picking the wrong channel can yield inaccurate results.

\subsection{A General Scenario}

In the experiments in this section we show that the $k$-sigma method produces accurate distance estimations also in the general case, i.e., when no assumptions are made on whether nodes are in LoS of each other, or not. First, we use all our data sets to estimate the constants $t_{\text {proc }}$ and $k$ using leave-one-out crossvalidation. Figure 8 depicts the results. We note the following two things: some 
estimations are not very accurate, and the estimations on the los-out data set are worse than those presented in Figure 6 . The reason for the latter is that we, in Figure 6, only use measurements from a single environment, causing the $k$-sigma method to optimize for that environment. Here, on the other hand, we have incorporated measurements from several different environments to optimize the $k$-sigma method for the general case.

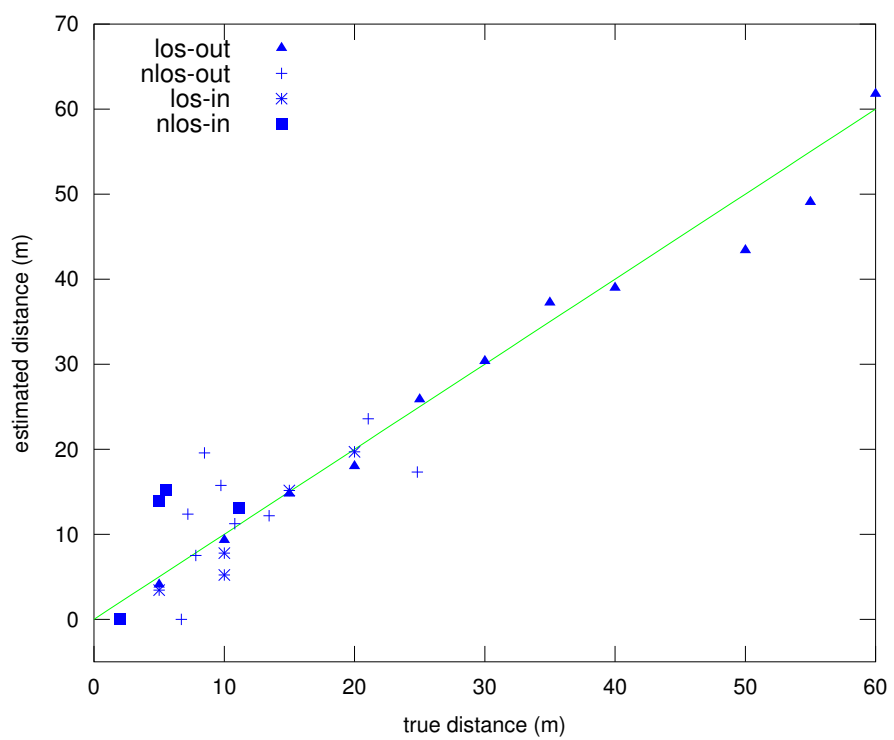

Fig. 8. Distance estimation with the $k$-sigma method in the general case. The method obtains accurate estimations also when nodes are not in LoS. Labels indicate to which data set measurements belong.

Regarding the first observation above, Figure 9 shows a comparison between the method proposed by Mazomenos et al. and the $k$-sigma method, both using the same measurements and hence also the same stable clock, i.e., only the methods themselves differ. For their method, we have selected the channel resulting in the smallest ToF measurements as described in 5.3. We obtain improvements for all data sets, and an overall improvement of 30\%. The overall RMS errors are 4.5 meter, and 6.4 meter for our method and the one proposed by Mazomenos et al., respectively. We also see that the $k$-sigma method outperforms their method for the two non-LoS data sets nlos-in and nlos-out. This is because the ToF measurements in these data sets are very inaccurate, and the $k$-sigma method is often able to remedy this, while the method used by Mazomenos et al. is not. The $k$-sigma method does this by exploiting the statistical relation between the inter-channel standard deviation of the ToF measurements, and the measurement error due to multi-path propagation, to estimate appropriate values of $t_{o f f}$ as explained in Section 4. 


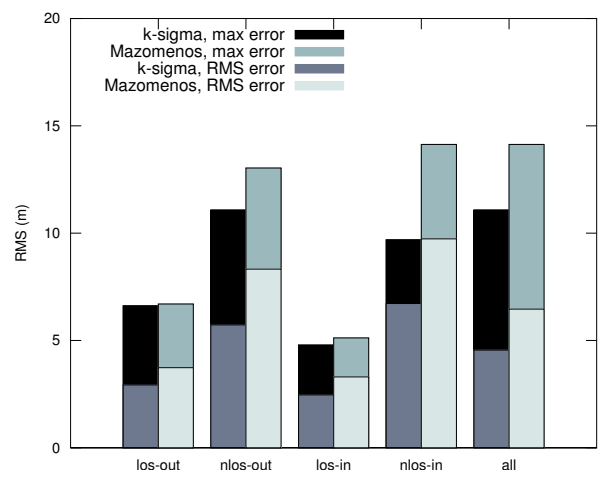

Fig. 9. Comparison between the k-sigma method and the one used by Mazomenos et al. using the same data. The $k$-sigma method obtains lower maximum errors, lower RMS errors for all different data sets, and an overall improvement of $30 \%$.

We perform a second experiment to evaluate how well the $k$-sigma method performs in environments from which no data have been available during calibration. Instead of leaving out one single measurement, we leave out whole data sets, one at a time, and use that set to evaluate the method. The results are given as RMS error in Table 1, which also shows the corresponding values for the $k$-sigma method from Figure 9, for comparison purposes.

\begin{tabular}{ccccc}
\hline Evaluation method & los-out & nlos-out & los-in & nlos-in \\
\hline leave-set-out & 3.1 & 5.4 & 2.6 & 7.0 \\
leave-one-out & 2.9 & 5.7 & 2.5 & 6.7 \\
\hline
\end{tabular}

Table 1. RMS errors (in meters) for the experiment where whole data sets are left out together with the results previously presented for the $k$-sigma method in Figure 9.

The experiments show that in our scenarios, the $k$-sigma method obtains accurate estimations even in environments from which no data has been used in the calibration phase to determine the parameters of the $k$-sigma method. The results show, however, that using data collected from the deployment environment provides more accurate estimations.

\subsection{Estimating the Accuracy}

Although we can obtain improved results using the $k$-sigma method, we yield inaccurate results in some scenarios. From Figure 9 we see that we obtain a maximum error of 11 meters. Given this imperfection, information of the expected 
estimation accuracy can be valuable. This information can be used in localization algorithms to, e.g., weight measurements according to their estimated accuracy.

Under non-LoS conditions, the multi-path effect can be substantial which can lead to longer ToF measurements than required for the actual distance between sender and receiver. The fact that different channels can yield different propagation paths suggests that it is possible to use the variance of the ToF between the channels to determine the estimation accuracy.

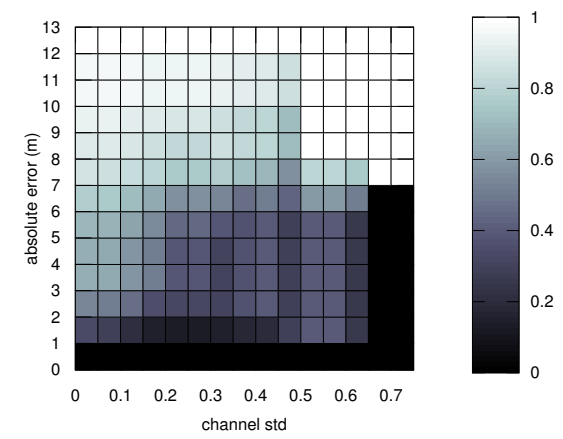

Fig. 10. The color at point $(\mathrm{x}, \mathrm{y})$ indicates the probability of having an absolute error less than y meters, given that the standard deviation for the measurements from different channels is greater than $\mathrm{x}$. The probability for high errors increases with the standard deviation. We believe that the abrupt change around $x=0.5$ is an artifact of having few samples with a standard deviation above this value.

We use our data sets to approximate the cumulative probability distribution for the measurement error given a lower limit for the inter-channel standard deviation. That is, $P(|e| \leq y \mid \sigma \geq x)$, where $|e|$ is the absolute measurement error, and $\sigma$ the inter-channel standard deviation. The approximated distribution is shown in Figure 10. According to the figure, it is more probable to have a high accuracy if the channel standard deviation is low while a high channel standard deviation indicates low accuracy. This suggests that the standard deviation can be used to determine the estimation accuracy.

\section{Related Work}

Many different localization measurement techniques have been proposed $[6,12$, 13,17]. Most of them only work under quite strict assumptions about the environment. Examples of such assumptions are that nodes are positioned in LoS of each other [20], and the existence of special, tightly synchronized anchor nodes [21]. Moreover, many solutions require additional hardware, e.g., ultra sound transceivers [16] or special directional antennas [15].

One technique that does not require specialized hardware, nor strict assumptions regarding the environment is RF based two-way ToF as proposed by Ma- 
zomenos et al. [14]. Inspired by Mazomenos' scheme, we also implement a twoway ranging scheme but improve it in several aspects as discussed in the paper.

There are previous efforts using ToF to estimate distances in wireless sensor networks, for example, by Lanzisera et al. [10], Pichler et al. [18] and Karalar and Rabaey [9]. All these efforts rely on special hardware in terms of FPGAs for advanced signal processing or the simultaneous reception of signals on several channels. Localization using ToF is more accurate with UWB radios due to the physical properties of UWB signals, in particular the large bandwidth. Therefore, there are a number of approaches using UWB-based positioning including those by Gezici et al. [5] and Alsindi et al. [2]. Alcock et al. have also combined UWB positioning and MAC layer communication [1].

Similar to our work, Bardella et al. exploit multichannel transmissions to reduce the negative effect of multi-path on distance estimation [3]. In contrast to us, they focus on RSSI-based distance estimation rather than ToF.

There are other studies on localization that have considered non LoS signals. $\mathrm{Li}$ has proposed a model for angle-of-arrival estimation for mobile phones that requires a sufficient number of base stations [11]. Similar to Li and many others, Venkatraman el al. [22] also target mobile phone systems rather than resourceconstrained wireless sensor networks.

\section{Conclusions}

In this paper we have presented a two-way time-of-flight ranging method for distance estimation in sensor networks. Our main contribution is that we show that by using multiple channels we also achieve good estimates when nodes are not placed in line-of-sight.

\section{Acknowledgements}

This work has been partly performed within the SICS Center for Networked Systems funded by VINNOVA, SSF, KKS, ABB, Ericsson, Saab SDS, TeliaSonera, T2Data, Vendolocus and Peerialism and has been partially supported by the FP7 NoE CONET. Thanks to Evangelos Mazomenos for making his data available.

\section{References}

1. P. Alcock, U. Roedig, and M. Hazas. Combining Positioning and Communication Using UWB Transceivers. In Proceedings of the International Conference on Distributed Computing in Sensor Systems, Marina del Rey, USA, June 2009.

2. N. A. Alsindi, B. Alavi, and K. Pahlavan. Measurement and modeling of ultrawideband TOA-based ranging in indoor multipath environments. IEEE Transactions on Vehicular Technology, 58(3):1046-1058, March 2009. 
3. A. Bardella, N. Bui, A. Zanella, and M. Zorzi. An experimental study on ieee 802.15. 4 multichannel transmission to improve rssi-based service performance. In REALWSN 2010, Colombo, Sri Lanka, December 2010.

4. J. Elson and K. Römer. Wireless sensor networks: A new regime for time synchronization. ACM SIGCOMM CCR, 33(1):149-154, 2003.

5. S. Gezici et al. Localization via ultra-wideband radios: a look at positioning aspects for future sensor networks. Signal Processing Magazine, IEEE, 22(4):70-84, 2005.

6. T. He, C. Huang, B.M. Blum, J.A. Stankovic, and T. Abdelzaher. Range-free localization schemes for large scale sensor networks. In ACM MobiCom, San Diego, USA, September 2003.

7. Texas Instruments. $2.4 \mathrm{ghz}$ ieee 802.15.4 / zigbee-ready rf transceiver (rev. b), March 2007. CC2420 datasheet.

8. Texas Instruments. MSP430x2xx family user's guide (rev. H), December 2010.

9. T.C. Karalar and J. Rabaey. An rf tof based ranging implementation for sensor networks. In IEEE ICC, Istanbul, Turkey, June 2006.

10. S. Lanzisera, D.T. Lin, and K.S.J. Pister. Rf time of flight ranging for wireless sensor network localization. In International Workshop on Intelligent Solutions in Embedded Systems, June 2006.

11. X. Li. A selective model to suppress nlos signals in angle-of-arrival (aoa) location estimation. In PIMR, Boston, USA, 1998.

12. G. Mao, B. Fidan, and B. Anderson. Wireless sensor network localization techniques. Computer Networks, 51(10):2529-2553, 2007.

13. M. Maróti, P. Völgyesi, S. Dóra, B. Kusỳ, A. Nádas, Á. Lédeczi, G. Balogh, and K. Molnár. Radio interferometric geolocation. In Proceedings of the 3rd international conference on Embedded networked sensor systems (ACM SenSys), 2005.

14. E. Mazomenos, D. De Jager, J. Reeve, and N. White. A two-way time of flight ranging scheme for wireless sensor networks. In European Conference on Wireless Sensor Networks, Bonn, Germany, February 2011.

15. A. Nasipuri and K. Li. A directionality based location discovery scheme for wireless sensor networks. In WSNA'02, Atlanta, USA, September 2002.

16. G. Oberholzer, P. Sommer, and R. Wattenhofer. Spiderbat: Augmenting wireless sensor networks with distance and angle information. In International Conference on Information Processing in Sensor Networks (IPSN), April 2011.

17. N. Patwari, J.N. Ash, S. Kyperountas, A.O. Hero III, R.L. Moses, and N.S. Correal. Locating the nodes: cooperative localization in wireless sensor networks. Signal Processing Magazine, IEEE, 22(4):54-69, 2005.

18. M. Pichler, S. Schwarzer, A. Stelzer, and M. Vossiek. Multi-channel distance measurement with ieee 802.15. 4 (zigbee) devices. IEEE JSAC, 3(5):845-859, 2009.

19. K.L. Priddy and P.E. Keller. Artificial neural networks: an introduction, volume 68. 2005.

20. K. Römer. The lighthouse location system for smart dust. In Proceedings of the 1st international conference on Mobile systems, applications and services (ACM MobiSys), San Francisco, USA, May 2003.

21. C. Savarese, J.M. Rabaey, and J. Beutel. Location in distributed ad-hoc wireless sensor networks. In IEEE International Conference on Acoustics, Speech, and Signal Processing, Salt Lake City, USA, May 2001.

22. S. Venkatraman, J. Caffery Jr, and H.R. You. A novel toa location algorithm using los range estimation for nlos environments. Vehicular Technology, IEEE Transactions on, 53(5):1515-1524, 2004.

23. Zolertia. Z1 datasheet (rev. c), March 2010. Zolertia Z1 datasheet. 